\section{Cuidados familiares prestados por los ancianos del ámbito rural a distintas generaciones}

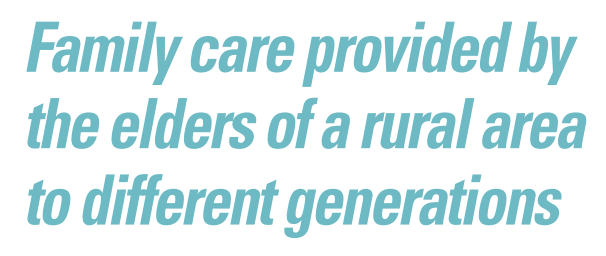

\author{
Encarnación Rubio Aranda' ${ }^{1}$ \\ Magdalena Comín Comín² \\ Gema Montón Blasco ${ }^{3}$ \\ Tomás Martínez Terrer ${ }^{4}$ \\ Rosa Magallón Botaya ${ }^{5}$
}

1. Facultad de Medicina. Universidad de Zaragoza.

2. Facultad Universitaria de Ciencias de la Salud. Universidad de Zaragoza.

3. Servicio de Enfermería de la Salud. Zaragoza.

4. Facultad de Medicina. Universidad de Zaragoza.

5. Salud. redlAPP (Carlos III 06/018). Instituto Aragonés de Ciencias de la Salud.

\section{RESUMEN}

Objetivos: Describir el perfil del cuidador mayor del ámbito rural, analizar las diferencias sociodemográficas, de salud, relaciones sociales y necesidades de servicios no cubiertas entre cuidadores familiares y no cuidadores.

Metodología: Se encuestaron 787 mayores de 64 años no institucionalizados pertenecientes a una Zona Básica de Salud de Zaragoza. Las variables recogidas mediante el cuestionario OARS-MAFQ

(OARS) fueron: sociodemográficas, necesidad de servicios, recursos sociales, económicos y salud física. Otras variables: capacidad funcional para las actividades de la vida diaria, básicas e instrumentales, índice de masa corporal y realización de actividad física.

Resultados: Los cuidadores de hijos/nietos fueron independientes para las actividades de la vida diaria, realizaron más actividad física, presentaron buena salud física y así lo percibieron. Los no cuidadores tuvieron mayor

dependencia y depresión. Los tres grupos mostraron buenos recursos sociales y económicos, aunque significativamente mejores los cuidadores de hijos/nietos, cuyas necesidades fueron: transporte público y viajar. Los no cuidadores refirieron mayor necesidad de ayuda para bañarse, vestirse, realizar tareas de casa y preparar comidas. Los cuidadores de cónyuges/padres necesitaron más tratamientos por problemas familiares y personales. Todos precisaron cuidados de enfermería.

Conclusiones: El perfil, características y necesidades del cuidador y no cuidador fueron diferentes, coincidiendo en la elevada necesidad de cuidados de enfermería.

PALABRAS CLAVE: cuidador familiar, anciano, nieto, salud, necesidades de servicios.

\section{Correspondencia:}

Encarnación Rubio Aranda

Facultad de Medicina, edificio A

c/ Domingo Miral s/n

50009 Zaragoza

Tel.: +34 976762703

E-mail: erubio@unizar.es

\title{
ABSTRACT
}

Objectives: To describe the profile of caregivers higher in rural areas, to analyze sociodemographic differences in health and social relations between family caregivers and non-caregivers, and service needs unmet. Methodology: We surveyed 787 people over 64 noninstitutionalized belonging to the Basic Health Area of Zaragoza. Variables were collected through the questionnaire OARS-MAFQ (OARS): sociodemographic, need for services, assessment of social, economic and physical health. Other variables were: functional capacity for activities of daily living, basic and instrumental, body mass index and physical activity undertaken.

Results: All caregivers of children/grandchildren were independent for activities of daily living, performed more physically active, had good physical and so perceived. Noncaregivers had the highest proportion of dependence and depression. The three groups had good social and economic resources, although significantly higher in those who care for children/grandchildren in which needs were perceived as more public transport and travel more. Non-caregivers reported more need for help with bathing, dressing, household chores and meal preparation. Caregivers of spouses/parents needed more treatment to personal or family problems. There was a great need for nursing care in the three groups.

Conclusions: The profile, characteristics and needs of caregiver and no-caregiver were different, coinciding only in high need for nursing care.

KEYWORDS: family caregivers, elderly, grandchildren, health, service needs.

\section{- INTRODUCCIÓN}

En nuestra sociedad, la familia desempeña una función de enlace entre generaciones, ya que no solo la forman los padres y los hijos, sino también los abuelos, tíos y otros parientes, todos los cuales actúan como una red de solidaridad y apoyo. Los vínculos multigeneracionales de la familia han sido considerados una fuente importante de apoyo, tanto para las generaciones mayores como las menores, y aunque por el envejecimiento poblacional los cuidados a personas mayores son los que más atención han recibido, no hay que olvidar que los abuelos son un punto de apoyo muy importante en el cuidado de los niños en las familias europeas ${ }^{1}$. 
Por apoyo y cuidado se entiende un conjunto de actividades orientadas a proporcionar bienestar físico, psíquico y emocional a las personas ${ }^{2}$. En el dictamen de Cohen y Eisdorfer citado por Lurdes y Storani en Netto $^{3}$, la motivación para cuidar a alguien se basa en cuatro razones principales: amor, gratitud, moralidad y voluntad propia de cuidar.

Para Chappell y Blandford ${ }^{4}$ las redes de soporte de cuidados se clasifican en formales e informales y tienen como fin la ayuda a las personas mayores. La red de soporte formal está compuesta por organismos de ayuda de servicios gubernamentales, mientras que la red informal (o red natural de ayuda) está constituida por el compañero, parientes, amigos y vecinos, aunque la familia sigue siendo el grupo social básico donde se intercambia el cuidado a personas en el hogar, tanto a la adulta como a la más joven. Estas redes prestan apoyo psicológico, relacionado con el bienestar, y apoyo instrumental, es decir, la ayuda física en momentos en que el anciano presenta una deficiencia en sus capacidades funcionales o una pérdida de autonomía, temporal o permanente ${ }^{5}$.

Actualmente, los cambios socioculturales y demográficos junto a la crisis del Estado de Bienestar hacen que, el cuidado, que ha estado siempre dentro del hogar, se esté reestructurando, por distintas causas y en todos los ámbitos. Un aumento de la demanda de cuidados y una disminución del número de personas dispuestas a realizarlos de forma desinteresada, incluso dentro de la familia, está determinando que los cuidados que se prestan a los ancianos y por los ancianos sean mayores. Por ello, conocer la situación, necesidades y demandas de las personas que están haciendo en la actualidad esta función de cuidado se considera, desde las instituciones públicas, el primer paso para poner en marcha medidas de apoyo a los cuidadores informales. Está claro que, para poder cumplir los desafíos y demandas que supone cuidar, precisan apoyo y acceso a recursos comunitarios que los ayuden a sobrellevar los problemas que surgen de su particular situación y a cuidar su propia salud y bienestar.

Numerosas investigaciones se han realizado en los últimos años en España y en otros países; sin embargo, la mayoría se centran en el análisis del cuidador de personas mayores, sobre todo en población con demen$\mathrm{cia}^{6-10}$. Pocos estudios se han dedicado al estudio conjunto del cuidador de familiares mayores y de otros miembros de su familia, incluidos los nietos, así como a describir sus necesidades de servicios ${ }^{11}$. En esta línea, nos planteamos como objetivos: describir el perfil del cuidador mayor del ámbito rural, analizar las diferencias sociodemográficas, en salud y de relaciones sociales entre los cuidadores familiares y no cuidadores, así como las necesidades de servicios no cubiertas.

\section{- METODOLOGÍA}

Población y muestra. Estudio transversal de todas las personas mayores de 64 años, no institucionalizadas, pertenecientes a la Zona Básica de Salud de Casetas (Zaragoza). Esta zona forma parte del Área de Salud 3 de Zaragoza.

Son criterios de inclusión: tener 65 años o más, estar en posesión de la Tarjeta Sanitaria en diciembre de 2008 y firmar un consentimiento informado de participación en el estudio. Se excluyeron las personas que no se encontraron en su domicilio en tres ocasiones diferentes durante el periodo de recogida de datos y los que tuvieron más de siete fallos en el cuestionario de Pfeiffer. La población diana ascendió a 1299 personas. Desde enero a diciembre de 2009 se procedió a realizar la entrevista personal en los domicilios. Tras las pérdidas ocasionadas en distintos momentos de la selección, el estudio se realizó a 787 personas (fig. 1).

Definición de variables e instrumentos de medida. La variable principal fue ¡es usted cuidador y de quién cuida?, de respuesta tricotómica: "no cuidador", "cónyuge/padres", "hijos/nietos". Como variables expli-

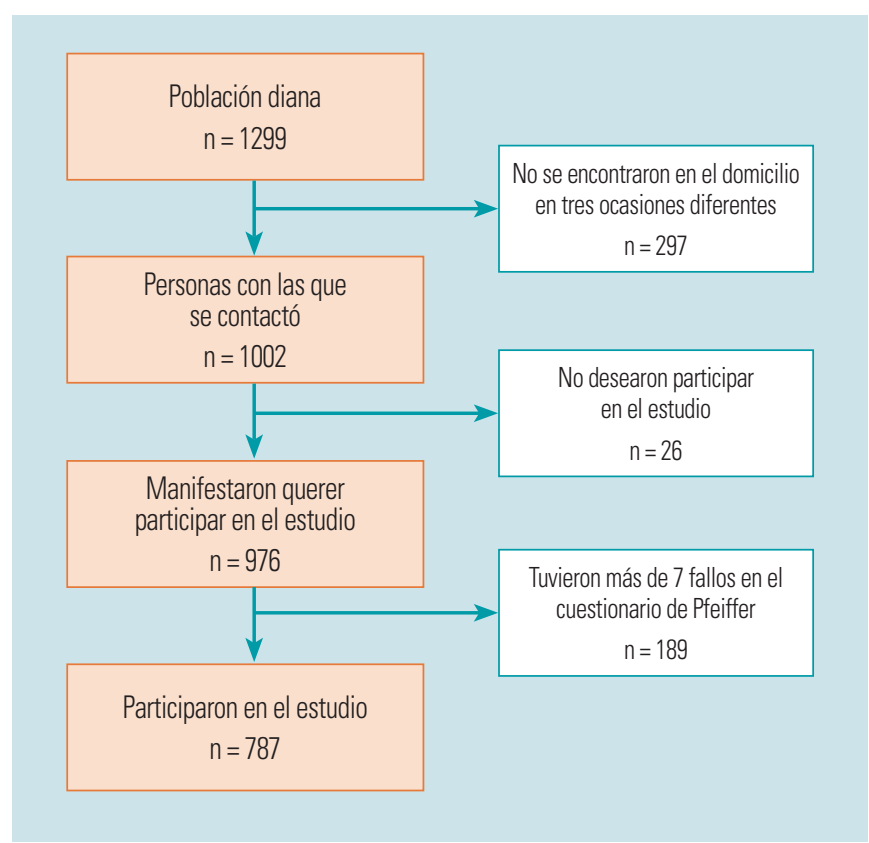

Figura 1. Diagrama de flujo de la selección de la muestra del estudio.

cativas se valoraron: realización de actividad física y las recogidas por el cuestionario OARS-MAFQ (OARS) en su versión validada y adaptada al castellano ${ }^{12}$ : sociodemográficas, necesidad de servicios, valoración de recursos sociales, económicos y salud física. La salud física se valoró mediante 19 preguntas del cuestionario, una de las cuales interroga sobre 28 enfermedades crónicas más prevalentes en personas ancianas (como depresión, hipertensión, etc.); otra sobre el consumo de 18 grupos de medicamentos, y otra sobre el uso de 12 dispositivos de apoyo y prótesis. Los recursos sociales y económicos se obtuvieron tras valorar 9 y 14 cuestiones diferentes, respectivamente. Estas tres dimensiones: salud física, recursos sociales y económicos fueron evaluadas por el entrevistador, que les otorgó una puntuación en una escala de 6 puntos, donde 1 corresponde a excelente y 6 a totalmente deficiente. En el estudio que nos ocupa, esta escala fue recodificada para cada una de las dimensiones en: "bueno" si puntuaba 1 o 2 y "regular/malo" para el resto.

La capacidad funcional para las actividades básicas de la vida diaria $(A B V D)$ se clasificó según puntuación del test de Barthel ${ }^{13}$ en "dependiente" $(\leq 45)$ e "independiente" $(>45)$. La capacidad funcional para las actividades instrumentales de la vida diaria (AIVD) según el test de Lawton-Brody ${ }^{14}$, en independiente $(\leq 4)$ y dependiente $(>4)$.

La edad se recodificó en "menores de 75 años" y "75 años o más". El nivel de estudios, en "primaria sin terminar" y "primaria o más". El estado civil, en "soltero/divorciado", "casado" y "viudo". La salud autopercibida, como "buena" y "regular/mala". El índice de masa corporal (IMC), en "normopeso" (IMC $\leq 25)$ y "sobrepeso" (IMC > 25). El resto de las variables se dicotomizaron en "sí/no".

Análisis estadístico. Mediante test de la $\chi^{2}$ se obtuvo la relación entre la variable principal y las explicativas. El análisis de residuos tipificados de Haberman objetivó qué categorías de las variables estaban relacionadas. Nivel de significación exigido: $p<0,05$. Se utilizó la corrección de Bonferroni en caso de que la tabla fuera mayor de $2 \times 2$. Programa estadístico utilizado: SPSS 19.0.

Este estudio forma parte de un proyecto más amplio, aprobado por el Comité Ético de la Diputación General de Aragón (11/2009). El consentimiento informado se obtuvo de todos los participantes de acuerdo a los principios de la Declaración de Helsinki. 


\section{- RESULTADOS}

De los 787 encuestados, 177 (22,5\%) dijeron ser cuidadores; de ellos, 77 (43,5\%) cuidaban del cónyuge/padres/hermanos y 100 (56,5\%) de hijos/nietos.

Al comparar la variable principal con las sociodemográficas (tabla 1), se observó que en los tres grupos, el mayor porcentaje corresponde a mujeres; sin embargo, la proporción de hombres cuidadores de hijos/ nietos es significativamente mayor que en los otros dos grupos. Del mismo modo, en este grupo se encontraron en mayor proporción los menores de 75 ańos, con estudios primarios terminados. Aunque la mayoría de los encuestados estaban casados, este estado civil se dio en mayor proporción entre los que cuidaban de cónyuge/padres.

Más del 90\% de los entrevistados (tabla 1) en cada una de las categorías de cuidador dijeron tener alguna persona en quien confiar y creían que si estuvieran enfermos alguien les ayudaría. Los no cuidadores y los que cuidaban de cónyuge/padres se encontraban solos en mayor proporción que los cuidadores de hijos/nietos. En general, para los tres grupos analizados, los recursos sociales y económicos fueron buenos, aunque significativamente mejores en los cuidadores de hijos/nietos.
La mayor proporción de diagnosticados de depresión se encontró entre los no cuidadores. Los cuidadores de hijos/nietos fueron los que en proporción significativamente mayor realizaban actividad física, tenían buena salud física y, además, así lo percibían.

Al valorar la capacidad funcional para las AVD, tanto básicas como instrumentales, el $100 \%$ de los cuidadores de hijos/nietos fueron independientes para ambas, con un mayor porcentaje de dependientes entre los no cuidadores.

Las necesidades percibidas (tabla 2) para los cuidadores de hijos/nietos fueron más transporte público y viajar; para los no cuidadores, más ayuda para bańarse o vestirse, realizar tareas de casa y preparación de comida, y para los cuidadores de cónyuges/padres más tratamiento por problemas familiares o personales. En los tres grupos existió una elevada necesidad de cuidados de enfermería.

\section{- DISCUSIÓN}

En esta investigación se encontró que las características del anciano que no cuida y el cuidador fueron diferentes. El no cuidador presentó una edad superior al resto, en muchas ocasiones vive solo (31\% viudo) y necesita ser cuidado, lo que probablemente hace que se sienta mal, tenga

Tabla 1. Tipo de cuidador y características demográficas y de salud

\begin{tabular}{|c|c|c|c|c|c|}
\hline & \multicolumn{4}{|c|}{ ¿Es usted cuidador y de quién cuida? } \\
\hline & & $\begin{array}{l}\text { No } \\
\text { n (\%) }\end{array}$ & $\begin{array}{c}\text { Cónyuge/padres } \\
\text { n (\%) }\end{array}$ & $\begin{array}{l}\text { Hijos/nietos } \\
\text { n (\%) }\end{array}$ & $p$ \\
\hline Sexo & mujer & $320(52,2)$ & $52(67,5)$ & $60(60,0)$ & 0,024 \\
\hline Edad & 65-74 años & $252(41,3)$ & $37(48,1)$ & $68(68,0)$ & $<0,0001$ \\
\hline \multirow{3}{*}{ Estado civil } & soltero/divorciado & $47(7,7)$ & $2(2,6)$ & $2(2,0)$ & \multirow{3}{*}{$<0,0001$} \\
\hline & casado & $371(60,8)$ & $74(96,1)$ & $80(80,0)$ & \\
\hline & viudo & $192(31,5)$ & $1(1,3)$ & $18(18,0)$ & \\
\hline Estudios & primaria o más & $236(38,7)$ & $44(57,1)$ & $64(64,0)$ & $<0,0001$ \\
\hline Recursos económicos & buenos & $418(68,5)$ & $57(74,0)$ & $89(89,0)$ & $<0,0001$ \\
\hline Tiene en quien confiar & sí & $572(93,8)$ & $76(98,7)$ & $97(97,0)$ & NS \\
\hline Alguien le ayudaría si estuviera enfermo & sí & $594(97,4)$ & $76(98,7)$ & $90(90,0)$ & $<0,0001$ \\
\hline Se encuentra solo a menudo & sí & $296(48,5)$ & $32(41,6)$ & $29(29,0)$ & $=0,001$ \\
\hline${ }^{*}$ Recursos sociales & buenos & $421(69,0)$ & $52(67,5)$ & $97(97,0)$ & $<0,0001$ \\
\hline Tiene depresión & sí & $95(48,5)$ & $18(23,4)$ & $10(10,0)$ & $=0,032$ \\
\hline IMC & normopeso & $320(52,5)$ & $34(44,2)$ & $54(54,0)$ & NS \\
\hline Realiza actividad física & sí & $231(37,9)$ & $20(26,0)$ & $65(65,0)$ & $<0,0001$ \\
\hline Salud percibida & buena & $275(45,1)$ & $36(46,8)$ & $71(71,0)$ & $<0,0001$ \\
\hline Salud física & buena & $389(63,8)$ & $57(74,0)$ & $83(83,0)$ & $<0,0001$ \\
\hline CF. ABVD & independiente & $586(91,6)$ & $76(98,7)$ & $100(100)$ & - \\
\hline CF. AIVD & independiente & $487(79,8)$ & $76(98,7)$ & $100(100)$ & - \\
\hline
\end{tabular}

ABVD: actividades básicas de la vida diaria; AIVD: actividades instrumentales de la vida diaria; CF: capacidad funcional; IMC: índice de masa corporal; NS: no significativo. 
Tabla 2. Tipo de cuidador y necesidades percibidas

\begin{tabular}{|c|c|c|c|c|c|}
\hline \multirow{2}{*}{\multicolumn{2}{|c|}{ Necesita: }} & \multicolumn{4}{|c|}{ ¿Es usted cuidador y de quién cuida? } \\
\hline & & \multirow{2}{*}{$\begin{array}{c}\text { No } \\
\text { n }(\%) \\
306(50,2)\end{array}$} & \multirow{2}{*}{$\begin{array}{c}\text { Cónyuge/padres } \\
\text { n (\%) } \\
31(40,3)\end{array}$} & \multirow{2}{*}{$\begin{array}{c}\text { Hifos/nietos } \\
\text { n (\%) } \\
74(74,0)\end{array}$} & \multirow{2}{*}{$\begin{array}{c}p \\
<0,0001\end{array}$} \\
\hline Transporte más menudo & sí & & & & \\
\hline Practicar actividades recreativas & sí & $75(12,3)$ & $7(9,1)$ & $21(21,0)$ & 0,031 \\
\hline Viajar & sí & $220(36,1)$ & $35(45,5)$ & $62(62,0)$ & $<0,0001$ \\
\hline $\begin{array}{l}\text { Tratamiento por problemas familiares } 0 \\
\text { personales }\end{array}$ & sí & $53(8,7)$ & $15(19,5)$ & $3(3,0)$ & 0,001 \\
\hline Ayuda para vestirse, bañarse, etc. & sí & $153(25,1)$ & $8(10,4)$ & $12(12,0)$ & $<0,0001$ \\
\hline Cuidados de enfermería & sí & $435(71,3)$ & $56(72,7)$ & $69(69,0)$ & NS \\
\hline Ayuda en casa & sí & $375(61,5)$ & $40(51,9)$ & $35(35,0)$ & $<0,0001$ \\
\hline Ayuda para preparar la comida & sí & $281(46,1)$ & $21(27,3)$ & $20(20,0)$ & $<0,0001$ \\
\hline Asesoramiento legal & sí & $296(48,5)$ & $39(50,6)$ & $55(55,0)$ & NS \\
\hline
\end{tabular}

NS: no significativo.

depresión y presente con mayor frecuencia sentimiento de soledad. Los recursos económicos también fueron peores y las necesidades referidas se centraron en cubrir sus cuidados básicos en el aseo, alimentación y sobre todo para las tareas de la casa. Las dificultades económicas, físicas o psíquicas y la carencia de apoyo externo suficiente para el desarrollo de sus funciones obliga a los familiares, en muchas ocasiones, a renunciar a su papel de cuidadores, por su propia necesidad de ser cuidados, lo que puede justificar la alta prevalencia de necesidad de cuidados de enfermería mostrada por este colectivo.

El perfil del cuidador también fue distinto según el vínculo y la generación familiar que cuidaron. Así, en este trabajo, las características del cuidador del cónyuge/padres/hermanos coinciden con estudios realizados en España que se centran en el análisis del cuidador de otras personas mayores dependientes. El perfil es: mujer, con una edad media en la sexta década de vida y ama de casa, aunque también existe un grupo importante de cuidadores mayores de 65 ańos, generalmente cónyuges del enfermo, de especial importancia dada la gran comorbilidad que suelen presentar a esa edad ${ }^{15-17}$. Las personas mayores utilizan la ayuda familiar a medida que pierden la autonomía, y es un factor determinante de su mantenimiento en domicilio y supervivencia. Parece existir un orden preferencial por la familia frente a los amigos y el orden va desde el cónyuge a hijos y otros familiares, aunque para algunos autores las características de convivencia son más determinantes de la ayuda recibida que el tipo de vínculo ${ }^{4}$. Distintos estudios desde la perspectiva de género han seńalado que la mayoría de los cuidados recaen en las mujeres de la familia (hijas y esposas). En general, las mujeres son las que asumen entre sus responsabilidades el ocuparse de los demás por solidaridad intergeneracional y conyugal, como consecuencia de un proceso de socialización basado en la desigualdad de roles femeninos y masculinos ${ }^{18,19}$. Los datos obtenidos en esta investigación han puesto de manifiesto que hay diferencias de género, aunque las mujeres fueron en mayor porcentaje cuidadoras de cónyuges y hermanos, también cuidaron más a los nietos; sin embargo, en este caso se observó una mayor implicación del hombre, quizá debido a que en la actualidad los roles están menos establecidos y se trata de una población más joven.
Es notorio que cuando los cuidados fueron prestados al cónyuge y hermanos existieron peores recursos sociales, más depresión, peor percepción de su salud y un menor porcentaje de realización de actividad física respecto a los que cuidaron a los nietos. Está demostrado que el cuidado de familiares adultos con dependencia determina la aparición de distintos problemas físicos, psíquicos y sociofamiliares $^{7,9,20-22}$. Entre los elementos causales de la aparición de estos desórdenes cabe destacar la propia incapacidad del paciente anciano, las tareas que debe realizar el cuidador, la falta de rotación de los cuidados y de distribución de las tareas, y el impacto negativo de sus tareas sobre su calidad de vida ${ }^{23}$. Las consecuencias físicas suelen ser menos intensas que las psíquicas, aunque se dispone de pocos estudios específicamente centrados en las consecuencias del cuidado sobre la salud física; se describen, entre otras, un empobrecimiento en la autopercepción de salud y un grado variable de descuido en los hábitos de salud del cuidador, lo que coincide con los hallazgos de este estudio, ya que la mayoría de los cuidadores apenas realizaron actividad física y presentaron sobrepeso ${ }^{22}$. Entre los principales problemas psíquicos del cuidador se describe la depresión, cuya incidencia puede ser menor si existe apoyo social y sobre todo emocional de la familia (tener confidente $)^{24}$. Es de destacar que estos cuidadores, a pesar de presentar confidente en un $98,7 \%$ y buenas relaciones familiares, fueron los que en mayor porcentaje presentaron depresión y la necesidad de tratamiento por problemas familiares o personales. En las situaciones de discapacidad y, más aún, en los casos en que se alcanza una gran dependencia, se pueden presentar conflictos familiares por la sobrecarga en las ocupaciones y necesidades habituales que requiere la atención de estos $\operatorname{casos}^{25}$. En este sentido, los entrevistados, aun cuando se sintieron apoyados emocionalmente, suelen experimentar sentimientos ambiguos y contradictorios respecto del apoyo real recibido por parte de sus parientes, lo que asociado a una disminución de las actividades sociales y de ocio podría justificar estos hallazgos. Aunque los cuidadores refieran niveles altos de satisfacción acerca del relevo de cuidados, las evidencias sobre la reducción del estrés, sobrecarga o síntomas depresivos son discretas ${ }^{26}$. Por otro lado, este grupo refirió la necesidad de ayuda en casa y cuidado de enfermería, 
lo que podría estar relacionado con su peor situación funcional, la falta de información y el sentimiento de incapacidad para realizar los procedimientos relacionados con los cuidados ${ }^{27}$.

En publicaciones recientes ${ }^{1,28}$, aproximadamente un $42 \%$ de los mayores que cuidan a diario de sus nietos sufre ansiedad, estrés y tiene un sentimiento de frustración. Para la otra mitad, hacerse cargo de los nietos supone una tarea gratificante que les mantiene activos y con vitalidad. A muchos, esta actividad les permite tener un envejecimiento activo, con todo lo positivo que conlleva este término. Estudios transversales y longitudinales han examinado en abuelas que cuidan nietos los efectos sobre su salud, con resultados contradictorios ${ }^{29-31}$. En el contexto estudiado, el cuidado de nietos fue prestado por la población más joven, que presentó buena salud general lo que justificaría su mayor demanda de actividades recreativas, de viajar y de transporte. Según las conclusiones del estudio SHARE de $2006^{29}$, cuando existen dificultades físicas para realizar tareas, la incidencia del cuidado es siempre menor. Esta población también refirió con mayor frecuencia necesitar cuidados de enfermería, lo que podría deberse, junto a su mayor nivel educacional, al sentimiento de inseguridad, que al igual que los otros grupos, pueden presentar, al recaer sobre ellos la responsabilidad de los cuidados de la salud de sus nietos.

Como limitaciones de este estudio podemos definir las derivadas del diseño transversal del estudio, que no permitió establecer relaciones de causalidad. Además, con los datos de los que se dispone, no es posible determinar la frecuencia y el tipo de los cuidados prestados ni el grado de voluntariedad con la que los abuelos atienden.

Estos resultados ponen de manifiesto que las personas mayores que no cuidan son las que necesitan que les cuiden y que existen distintos perfiles de adultos cuidadores según esta actividad se dirija a familiares adultos o a nietos, lo que condiciona diferentes necesidades percibidas. Una necesidad referida en todos los grupos fue la de mayores cuidados de enfermería. La Atención Primaria es el marco idóneo a través del cual se debe intentar prevenir los distintos problemas y cubrir las necesidades del dependiente y del cuidador a través de una gestión individualizada por cada profesional y concretamente de mayor apoyo de enfermería. Se debe estimular la investigación longitudinal para poder identificar y ayudar a los mayores en mayor riesgo de necesidades no cubiertas

\section{- BIBLIOGRAFÍA}

1. Badenes Pla N, López López MT. Doble dependencia: abuelos que cuidan nietos en España. Colección Estudios y Monografías. Pamplona: Aranzadi; 2010

2. Curcio Borrero CL. Soporte social informal, salud y funcionalidad en el anciano. Hacia promoc. Salud. [Internet] 2008 [consultado 10 de julio de 2011];13(1). Disponible en: http://www.scielo.org.co/ scielo.php?pid=S0121-75772008000100004\&script=sci_arttext

3. Lurdes S, Storani M. Demência: Impacto para a Família e a Sociedade. En: Netto M, editor. Gerontologia: A Velhice e 0 Envelhecimento em Visão Globalizada. São Paulo: Atheneu; 1999 p. 146-59

4. Chappell N, Blandford A. Informal and formal care: Exploring the complementarity. Ageing Soc. 1991:11:299-317.

5. Puga D, Rosero -Bixby L, Glaser K, Castro T. Red social y salud del adulto mayor en perspectiva comparada: Costa Rica, España e Inglaterra. Población y Salud en Mesoamérica. 2007:5(1):1-21.

6. Esandi Larramendi N, Canga-Armayor A. Familia cuidadora y enfermedad de Alzheimer: una revisión bibliográfica. Gerokomos. 2011;22(2):56-61.

7. Fernández de Larrinoa Palacios P, Martínez Rodríguez S, Ortiz Marqués N, Carrasco Zabaleta M, Solabarrieta Eizaguirre J, Gómez Marroquín I. Autopercepción del estado de salud en familiares cuidadores y su relación con el nivel de sobrecarga. Psicothema. 2011;23(3):388-93

8. Lluch Bonet A, Morales López A, Cabrera Adán M, Betancourt Navarro M. Factores previsibles en la salud física y psicosocial del cuidador crucial del anciano con demencia en el hogar. Rev Cubana Enfermer (revista electrónica). 2010 [consultado 5 de julio de 2012];26(2):3-13. Disponible en: http://scielo.sld.cu/scielo. php? script=sci arttext\&pid=\$086403192010000200002\&ln$g=e s \& n r m=i s o \&$ tlng=es

9. Etters L, Goodall D, Harrison BE. Caregiver burden among dementia patient caregivers: a review of the literature. J Am Acad Nurse Pract. 2008;20(8):423-8.

10. Conde-Sala JL, Garre-Olmo J, Turró-Garriga O, López-Pousa
S, Vilalta-Franch J. Factors related to perceived quality of life in patients with Alzheimer's disease: the patient's perception compared with that of caregivers. Int J Geriatr Psychiatry. 2009; 24(6):585-94.

11. Kataoka-Yahiro MR. Filipino American grandparent caregivers roles, acculturation, and perceived health status. J Cult Divers. 2010;17(1):24-33

12. Grau G, Eiroa P, Cayuela A. Versión española del OARS Multidimensional Functional Assessment Questionnaire: adaptación transcultural y medida de la validez. Aten Primaria. 1996;17:486-95

13. Mahoney Fl, Barthel DW. Functional evaluation: The Barthel Index. Arch Phys Med Rehabil. 1965:14:61-5.

14. Lawton MP, Brody EM. Assessment of older people: self maintaining and instrumental activities of daily living. Gerontologist. 1969:9:179-86.

15. García-Calvente MM, Mateo-Rodríguez I, Maroto-Navarro G. El impacto de cuidar en la salud y calidad de vida de las mujeres. Gac Sanit. 2004;18 Suppl 2:83-92.

16. Rogero-García J. La distribución en España del cuidado formal e informal a las personas de 65 y más años en situación de dependencia. Rev Esp Salud Pública. 2009;83:393-405.

17. Hoffman F, Rodriguez R. Informal carers: who takes care of them? Policy Brief April 2010. Vienna: European Centre for Social Welfare Policy and Research; 2010.

18. Puyol R, Abellán A. Envejecimiento y dependencia. Una mirada al panorama futuro de la población española. Madrid: Mondial Assistance; 2006

19. Delicado Useres MV. Características sociodemográficas y motivación de las cuidadoras de personas dependientes. ¿Perfiles en transición? Revista Praxis Sociológica. 2006:6:200-34.

20. Segura Domínguez N, Gómez Caballo R, López Quintana R, Gil Paredes E, Saiz Cantero, C, Guevara JC. El anciano dependiente y el desgaste físico y psíquico de su cuidador. Rev Esp Geriatr Gerontol. 2006;41 Suppl 1:15-20.

21. Pinquart M, Sörensen S. Differences between caregivers and noncaregivers in psychological health and physical health: a meta-analysis. Psychol Aging. 2003;18(2):250-6

22. Schulz R, Sherwood PR. Physical and mental health effects of family caregiving. Am J Nurs. 2008;108 Suppl 9: 23-7.

23. Schoenmakers B, Buntix F, Delepeleire J. Factors determining the impact of care-giving on caregivers of elderly patients with dementia. A systematic literature review. Maturitas. 2010;66(2):191-200.

24. Domínguez JA, Ruíz, M, Gómez I, Gallego E, Valero J, Izquierdo MT. Ansiedad y depresión en cuidadores de pacientes dependientes. Semergen. 2012;38(1):16-23.

25. Esteban Gimeno AB, Mesa Lampré MP. Grado de tolerancia de los cuidadores ante los problemas de sus familiares con demencia. Rev Esp Geriatr Gerontol. 2008;43:146-53.

26. Goy E, Kansagara D, Freeman M. A systematic evidence review of interventions for non-professional caregivers of individuals with dementia. Evidence-based Synthesis Program (ESP) Center Portland VA Medical Center (libro electrónico) [consultado el 10 de julio de 2012]. Disponible en: http://www.ncbi.nlm.nih.gov/ books/NBK49194/pdf/TOC.pdf

27. Brito L. A saúde mental dos prestadores de cuidados a familiares idosos. Coimbra: Quarteto Editora; 2002

28. Guijarro Morales A. El Síndrome de la abuela esclava. Pandemia del siglo XXI. Granada: Grupo Editorial Universitario; 2001.

29. Survey of Health, Aging and Retirement in Europe (SHARE), Mannheim [consultado el 10 de julio de 2011]. Disponible en: http:// www.share-project.org/

30. Musil CM, Gordon NL, Warner CB, Zauszniewski JA, Standing T, Wykle M. Grandmothers and caregiving to grandchildren: continuity, change, and outcomes over 24 months. The Gerontologist 2011;51(1):86-100.

31. Kelley SJ, Whitley DM, Campos PE. Psychological distress in African American M, Grandmothers raising grandchildren: the contribution of child behavior problems, physical health, and family resources. Res Nurs Health. 2013;36(4):373-85 